
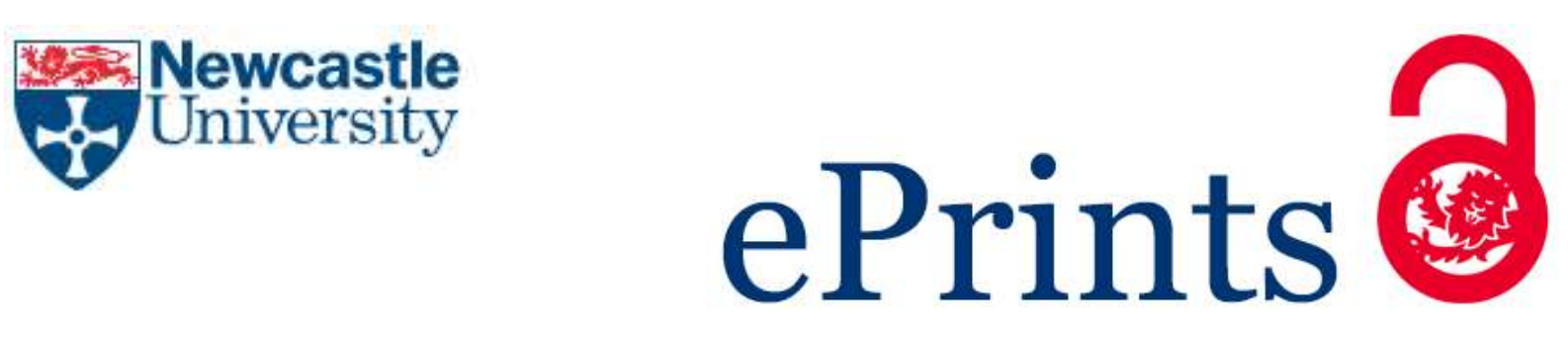

Pazos M, Peters K, Vollmer W. Robust peptidoglycan growth by dynamic and variable multi-protein complexes. Current Opinion in Microbiology 2017, 36, 55-61.

\title{
Copyright:
}

(c) 2017. This manuscript version is made available under the CC-BY-NC-ND 4.0 license

DOI link to article:

http://dx.doi.org/10.1016/i.mib.2017.01.006

Date deposited:

$28 / 02 / 2017$

Embargo release date:

15 February 2018

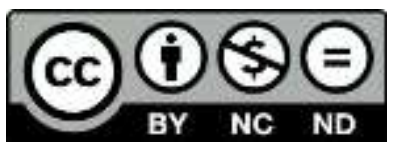

This work is licensed under a

Creative Commons Attribution-NonCommercial-NoDerivatives 4.0 International licence 


\section{Robust peptidoglycan growth by dynamic and variable multi-protein complexes}

2

3 Manuel Pazos, Katharina Peters and Waldemar Vollmer*

4

5 Centre for Bacterial Cell Biology, Institute for Cell and Molecular Biosciences, University of

6 Newcastle, Newcastle upon Tyne, United Kingdom, NE2 4HH

7

$8 *$ Correspondence to: Waldemar Vollmer, Institute for Cell and Molecular Biosciences, University of

9 Newcastle, Newcastle upon Tyne, United Kingdom, NE2 4HH; Email: w.vollmer@ncl.ac.uk; Tel:

$10 \quad+44(0) 1912083216$. 


\section{Abstract}

13 In Gram-negative bacteria such as Escherichia coli the peptidoglycan sacculus resides in the 14 periplasm, a compartment that experiences changes in $\mathrm{pH}$ value, osmolality, ion strength and other 15 parameters depending on the cell's environment. Hence, the cell needs robust peptidoglycan growth mechanisms to grow and divide under different conditions. Here we propose a model according to

17 which the cell achieves robust peptidoglycan growth by employing dynamic multi-protein complexes, 18 which assemble with variable composition from freely diffusing sets of peptidoglycan synthases, hydrolases and their regulators, whereby the composition of the active complexes depends on the cell cycle state - cell elongation or division - and the periplasmic growth conditions. 


\section{Introduction}

Bacteria surround their cytoplasmic membrane with a net-like layer made of peptidoglycan (PG), which consists of glycan chains connected by short peptides, to protect the cell from bursting while maintaining cell shape [1]. Gram-negative bacteria such as Escherichia coli have a mainly single layer of PG residing in the periplasm. The PG layer is elastic, expanding and shrinking in response to changes in the cell's turgor. It is fascinating that this thin PG layer with its essential, stabilizing role is constantly remodelled: an E. coli cell releases almost half of the material from the PG layer per generation in a process called PG turnover [2]. According to a model proposed by Höltje more than two decades ago [3], the high turnover is the consequence of a 3-for-1 PG growth mechanism involving the coordinated activities of synthetic and hydrolytic enzymes. Indeed, E. coli requires not only PG synthesis activities to expand its PG layer during growth and cell division, but also needs the activities of PG hydrolases for length growth and cell separation [4,5].

PG synthases are either glycosyltransferases (GTases), which polymerize the glycan strands, transpeptidases (TPases), which form peptide cross-links, or bi-functional enzymes which catalyze both reactions [6]. E. coli has three non-essential bi-functional GTase-TPases (PBP1A, PBP1B and PBP1C) but requires one of the major enzymes, PBP1A and PBP1B, for growth, while PBP1C is dispensable. PBP1A and PBP1B require the interaction with a cognate outer-membrane lipoprotein, LpoA and LpoB, respectively, for stimulation and functioning in the cell [7-10]. There is also a nonessential GTase (MtgA) and two TPases with specific and essential roles in cell elongation (PBP2) or division (PBP3). PBP2 and PBP3 are genetically and functionally linked to a member of the SEDS (shape, elongation, division and sporulation) family of integral membrane proteins (RodA and FtsW, respectively), for which different functions have been proposed. While biochemical evidence showed that FtsW and other SEDS proteins are capable of flipping the PG precursor lipid II and phospholipids across the membrane of proteoliposomes $[11,12]$, whole cell assays and genetic data were used to conclude that MurJ, and not RodA/FtsW, is a lipid II flippase [13]. The new crystal structure of MurJ supports a transport function but does not identify the ligand [14]. Based on genetic data and assays 
with a partially purified $B$. subtilis RodA fraction it was also suggested that SEDS proteins function as GTases and not as lipid II flippases [15]. However, E. coli FtsW did not show GTase activity but formed a trimeric complex with PBP3 and PBP1B and controlled lipid II polymerization of PBP1B [16]. Due to these conflicting conclusions made from biochemical assays and in vivo genetic data further experiments are needed to clarify the roles of MurJ and SEDS proteins.

E. coli has more than 20 PG hydrolases [lytic transglycosylases (LTs); $N$-acetylmuramyl-LAla amidases (amidases); endopeptidases (EPases); carboxypeptidases (CPases)] in its periplasm cleaving covalent bonds in the PG layer to release PG fragments (see Table 1 for enzyme names). It is not known how most of these hydrolases are regulated, but the uncontrolled activities of the autolysins (LTs, amidases and EPases) cause cell lysis under certain conditions, for example when PG synthesis is blocked by a $\beta$-lactam. Elegant work from the Bernhardt laboratory showed that EnvC and NlpD, which were originally thought to have PG hydrolase activity [17,18], are not enzymes but are needed to stimulate the activities of the PG amidases AmiA, AmiB and AmiC for septum PG cleavage and daughter cell separation [19].

In this opinion article, we present our current view on the mechanisms of PG synthesis in $E$. coli, with the particular aim to emphasize the dynamics of the process with respect to the assembly and disassembly of PG synthesis complexes that move along the PG layer to incorporate new PG and cleave the old material. We present a model according to which the conditions in the periplasm (such as $\mathrm{pH}$ value, osmolality, etc.), which are affected by the growth medium, dictate the composition of dynamically assembled PG synthesis complexes, ensuring that PG synthases and hydrolases are optimally active and regulated at any growth condition. This model explains why E. coli maintains large sets of seemingly redundant PG enzymes.

\section{Assembly and disassembly of dynamic PG synthesis complexes}

Theoretically, PG growth could be carried out from lipid II precursor by a minimal system of only three or four active domains (Figure 1) [20]. A GTase produces a glycan chain, which is attached to 
the existing PG layer by one or two TPases, while an endopeptidase (EPase) cleaves in the existing layer to allow the insertion of the new strand for surface expansion of the PG layer. A more complicated model, which explains PG turnover, assumes the incorporation of three glycan chains and the concomitant removal of one old strand (Höltje's 3-for-1 model) [3]. While the precise mechanism of PG growth remains unknown, these models are consistent with the observed large number of interactions detected for PG enzymes (reviewed in [6,21]), suggesting the existence of PG synthesizing multi-enzyme complexes. However, it became clear that several structural and regulatory proteins are involved to control the assembly and disassembly of these complexes and/or to position them in the cell envelope and control their activity [7].

The dynamic bacterial cytoskeleton regulates PG synthesis from inside the cell, presumably by facilitating the assembly, positioning and/or activation of the PG synthesis complexes [22-24]. During cell elongation the bacterial actin-like MreB and the associated proteins MreC, MreD and RodZ, which are essential to maintain the rod-shape of the cell, regulate PG synthesis complexes with PBP2 and PBP1A. MreB directly interacts with the main cell division protein, the tubulin-like FtsZ, and this interaction is important to initiate division [25]. During cell division, FtsZ and more than 9 associated essential cell division proteins, facilitate the formation of the ring-like division septum at mid-cell [26], including the synthesis of the septal PG catalyzed mainly by PBP3 and PBP1B [27,28]. Interestingly, recent work shows that in Bacillus subtilis and E. coli FtsZ moves in patches around the closing division ring by a GTP-dependent treadmilling mechanisms [22,29]. FtsZ movement drives a similar movement of the PG synthase PBP2B, the class B PBP essential for cell division in B. subtilis [22]. Hence, during cell division, the septal PG grows inwards by circular attachment of new material until the septum is closed.

During cell elongation MreB forms dynamic filamentous structures or foci that rotate around the short axis of the cell in the direction of the glycan chains [24]. The movement of these MreB foci depends on ongoing PG synthesis, which is in sharp contrast to the movement of FtsZ patches during cell division, which does not require PG synthesis [22]. Remarkably, MreB does not rotate in certain 
rod-shaped mutants of $E$. coli carrying an altered MreB and lacking RodZ, which is an cell elongation protein that couples MreB rotation with cell wall synthesis [23]. The rotation of MreB is not required to maintain rod-shape under standard laboratory condition, but is important for the robustness of shape-maintenance under conditions of cell wall stress [23]. Although the specific inactivation of PBP2 halts the rotation of the MreB foci [24], PBP2 molecules were reported to move much faster and erratic in a diffusive manner arguing against long-lived complexes containing MreB and PBP2 [30]. However, a more recent study identified sub-populations of PBP2 and the SEDS protein RodA that rotate with similar, slow velocity as MreB foci around the short axis of the cell, suggesting that these slower molecules of PBP2 and RodA move together in a complex with MreB (although colocalization has not been demonstrated) [31]. The rotation of MreB was independent of the activity of class A PBPs and, under these conditions, only fast moving, diffusive PBP1B molecules could be observed [31]. This diffusive, undirectional motion of PBP1A and PBP1B was confirmed in another study [32] which also identified sub-populations of fast and slow molecules of PBP1A and showed that the motion of PBP1A is modulated by an inhibitor (cefsulodin) and an activator (LpoA) [32]. However, it remains to be seen which of the sub-populations of PBP molecules are enganged in PG synthesis. PBP1A has been functionally linked to cell elongation by its cooperative interaction with PBP2 and side-wall localization [33]; hence, one would predict a yet unobserved sub-population of PBP1A that shows a similar dynamical behaviour as PBP2 during rounds of PG synthesis. Alternatively, class A and B PBPs act mostly independent of each other, interacting only sporadically with each other [31]. Overall, the available single molecule tracking data suggest that PG synthesis complexes are not static entities. Rather, functional complexes assemble from their components when PG synthesis is required, regulated by cytoskeletal elements, and they become fully activated by outer membrane anchored lipoproteins. After some time of active PG synthesis the complexes disassemble leaving the individual components to freely diffuse in the cell membrane or periplasm until the next round of PG synthesis is initiated. Rounds of dynamic assembly, synthesis activity and disassembly 
are also known from other complexes synthesizing macromolecules, such as the ribosome or the RNA polymerase, which have well characterized initiation, elongation and termination steps.

\section{Redundancy in PG synthesis and hydrolysis}

E. coli has more than 50 enzymes for PG synthesis and hydrolysis, and the enzymatic reactions take place in the cytoplasm, at the cytoplasmic membrane and in the periplasm [6]. Ten out of the twelve cytoplasmic and membrane-bound reactions leading to lipid II precursor are catalysed by single, essential enzymes (Mur enzymes and MraY), and only the D-alanine racemase and D-Ala-D-Ala ligase steps are catalyzed by two redundant enzymes. In sharp contrast, there is significantly more redundancy associated with the enzymatic steps that take place at the periplasmic side of the cytoplasmic membrane and in the periplasm itself. In fact, with the notable exception of the class B PBP - flippase (and/or GTase) pairs PBP2 - RodA and PBP3 - FtsW, which have specific roles in cell elongation and division, respectively, all other reactions are catalysed by (semi)-redundant enzymes (Table 1). This includes essential functions such as the GTase/TPase activities of the class A PBPs and their regulators, PBP1A - LpoA and PBP1B - LpoB, respectively. A double mutant lacking both class A PBPs (or both regulators) is not viable, but each of the single mutants is [34]. The single mutants have mild phenotypes under standard laboratory conditions, but severe ones under more stringent conditions (for example, [33,35]) suggesting that PBP1B - LpoB and PBP1A - LpoA are not completely interchangeable. This semi-redundancy is consistent with the greater enrichment of PBP1B at the cell division site and its interactions with the cell division proteins PBP3 and FtsN [27,36], and with members of the Tol-system, CpoB and TolA [37], suggesting a role in cell division. By contrast, PBP1A is more specialised in cell elongation based on its side-wall localization and interaction with PBP2 [33]. It is feasible to speculate that the semi-redundant PBP1A and PBP1B share some common interaction partners in the elongation and division complexes and with the Tolsystem. E. coli has two further, non-essential PG synthases, PBP1C and MtgA, whose functions are 
not well understood, although MtgA localizes to the division site and interacts with PBP3, FtsW and the cell division protein FtsN [38].

There are two other periplasmic PG synthesis reactions that both involve redundant, nonessential LD-TPase enzymes. YnhG and YcbB synthesize the rare LD-cross-links [39], and ErfK, YbiS and YcfS attach Braun's lipoprotein Lpp to the PG contributing to the tight linkage between the sacculus and the outer membrane to stabilize the cell envelope [40].

The enzyme redundancy is greatest in the case of the PG hydrolases that act on the PG sacculus. None of the 8 LTs, 6 EPases, 4 amidases or 7 CPases is essential, and many multiple mutant strains have no or mild phenotypes, making it difficult to define a clear role for each of these enzymes. Nonetheless, some of the hydrolases appear to have specialized functions. Three amidases (AmiA, $\mathrm{AmiB}$ and $\mathrm{AmiC}$ ) and their regulators (EnvC and NlpD) have redundant and significant roles in septum cleavage during cell division, and a mutant lacking all three enzymes grows in chains of unseparated cells [4]. Additional deletions of LTs and EPase encoding genes exacerbate the chaining phenotype, indicating that other hydrolases can participate in septum cleavage to partially compensate for the loss of amidases [41]. During cell elongation three EPases (MepS, MepM and MepH) have a redundant and essential role in cleaving cross-links in the sacculus for PG growth, such that inactivation of all three enzymes inhibits PG growth during cell elongation leading to cell lysis [5,42]. DD-CPase activity is not essential, but the collective pentapeptide-trimming activity of the 7 CPases is required to maintain normal rod-shape, presumably by regulating the amount and/or position of available pentapeptides for PBP-mediated cross-linking activity [43,44]. While single deletion of DD-CPase genes has no or only minor consequences, certain multiple mutants produce misshapen cells with enlarged diameter and even branches $[43,44]$. Besides their PG synthase activity, PBP1A and PBP1B also exhibit CPase activity at lower $\mathrm{pH}$ values and in the presence of their cognate Lpo activators [7]. Hence, the DD-CPases represent another example of a set of seemingly redundant PG enzymes. 
Why does E. coli maintain multiple, seemingly redundant PG enzymes for each reaction in the periplasm, while most cytoplasmic steps are catalysed by a single, essential enzyme? We hypothesize that the reason for the higher redundancy is that the properties and composition of the periplasm vary with changes in the cell's environment, while the cytoplasm is a less-changing, well-buffered differ in their activity ranges. without the need of having multiple enzymes for the same biosynthetic step. However, the periplasm is less buffered and significantly affected by the $\mathrm{pH}$ value, ion strength and osmolality of the growth medium, because the outer-membrane allows the entry of molecules with less than $\sim 600 \mathrm{Da}$ [45]. $E$. coli maintains robust growth at a range of conditions, for example at acidic $\mathrm{pH}$ or in the presence of high concentration of $\mathrm{NaCl}$, which can affect periplasmic processes such as the essential PG synthesis. Hence, we suggest that the redundancy of periplasmic PG enzymes has evolved to allow E. coli to grow at a wide range of growth conditions. The various PG enzymes have different properties and optimal conditions for activity, but the combined activities of the enzymes of each set (PBPs, TPases, LTs, EPases, amidases, CPases) may cover the whole range of conditions at which $E$. coli grows (Figure 2). In support of this view, we have recently found that one of the so-called 'minor' CPases, PBP6b, which is poorly expressed and has no known role at standard growth conditions, becomes important in E. coli cells growing at $\mathrm{pH} 5$ [46]. Under this condition, the PBP6b gene is more expressed, the produced protein is more stable and more active, and cells require PBP6b to maintain normal cell shape [46]. By contrast, when cells grow at near neutral pH the major DDCPase, PBP5 is more important. Another hydrolase with an activity optimum at non-standard conditions is the LT MltA, which is $\sim 10$ times more active at $30^{\circ} \mathrm{C}$ than at $37^{\circ} \mathrm{C}$ [47]. Although more work is needed to characterize the different PG enzymes at various conditions, these examples support the notion that $E$. coli achieves robust PG synthesis using sets of (seemingly redundant) enzymes that 


\section{Model for condition-dependent complex composition}

203 Taking into account the interactions of PG enzymes and their regulators, their cellular localization 204 and dynamics, and the multiplicity of PG enzymes in the periplasm, we propose a model according 205 other questions will surely keep us busy in the years to come.

\section{Acknowledgements}


This work was supported by the Wellcome Trust (101824/Z/13/Z), the MRC (MR/N002679/1) and European Commission (FP7-PEOPLE-2013-IEF-623868). We thank Alexander Egan for critical reading of the manuscript.

\section{References}

1. Vollmer W, Blanot D, de Pedro MA: Peptidoglycan structure and architecture. FEMS Microbiol Rev 2008, 32:149-167.

2. Goodell EW, Schwarz U: Release of cell-wall peptides into culture-medium by exponentially growing Escherichia coli. J Bacteriol 1985, 162:391-397.

3. Höltje JV: Growth of the stress-bearing and shape-maintaining murein sacculus of Escherichia coli. Microbiol Mol Biol Rev 1998, 62:181-203.

4. Heidrich C, Templin MF, Ursinus A, Merdanovic M, Berger J, Schwarz H, de Pedro MA, Höltje JV: Involvement of $\mathrm{N}$-acetylmuramyl-L-alanine amidases in cell separation and antibiotic-induced autolysis of Escherichia coli. Mol Microbiol 2001, 41:167-178.

5. Singh SK, SaiSree L, Amrutha RN, Reddy M: Three redundant murein endopeptidases catalyse an essential cleavage step in peptidoglycan synthesis of Escherichia coli K12. Mol Microbiol 2012, 86:1036-1051.

6. Typas A, Banzhaf M, Gross CA, Vollmer W: From the regulation of peptidoglycan synthesis to bacterial growth and morphology. Nat Rev Microbiol 2012, 10:123-136.

* 7. Egan AJ, Biboy J, van't Veer I, Breukink E, Vollmer W: Activities and regulation of peptidoglycan synthases. Philos Trans R Soc Lond B Biol Sci 2015, 370.

Comprehensive summary about the current knowledge on the interactions and regulation of PG synthases from E. coli. Shows for the first time the DD-CPase activity of stimulated class 
8. Markovski M, Bohrhunter JL, Lupoli TJ, Uehara T, Walker S, Kahne DE, Bernhardt TG: Cofactor bypass variants reveal a conformational control mechanism governing cell wall polymerase activity. Proc Natl Acad Sci U S A 2016, 113:4788-4793.

9. Paradis-Bleau C, Markovski M, Uehara T, Lupoli TJ, Walker S, Kahne DE, Bernhardt TG: Lipoprotein cofactors located in the outer membrane activate bacterial cell wall polymerases. Cell 2010, 143:1110-1120.

10. Typas A, Banzhaf M, van den Berg van Saparoea B, Verheul J, Biboy J, Nichols RJ, Zietek M, Beilharz K, Kannenberg K, von Rechenberg M, et al.: Regulation of peptidoglycan synthesis by outer-membrane proteins. Cell 2010, 143:1097-1109.

* 11. Mohammadi T, Sijbrandi R, Lutters M, Verheul J, Martin NI, den Blaauwen T, de Kruijff B, Breukink E: Specificity of the transport of lipid II by FtsW in Escherichia coli. J Biol Chem 2014, 289:14707-14718.

Provides biochemical evidence for the lipid II flippase activity of FtsW from E. coli reconstituted in proteoliposomes, whereas Reference 13 concludes from genetic data that MurJ is the lipid II flippase. Reference No. 15 provides genetic evidence and biochemical data with a partially purified RodA from $B$. subtilis and these authors suggest that SEDS proteins have PG glycosyltransferase activity.

12. Mohammadi T, van Dam V, Sijbrandi R, Vernet T, Zapun A, Bouhss A, Diepeveen-de Bruin M, Nguyen-Disteche M, de Kruijff B, Breukink E: Identification of FtsW as a transporter of lipid-linked cell wall precursors across the membrane. EMBO J 2011, 30:1425-1432.

* 13. Sham LT, Butler EK, Lebar MD, Kahne D, Bernhardt TG, Ruiz N: Bacterial cell wall. MurJ is the flippase of lipid-linked precursors for peptidoglycan biogenesis. Science 2014, 345:220-222.

See comment at Reference No. 11.

14. Kuk AC, Mashalidis EH, Lee SY: Crystal structure of the MOP flippase MurJ in an inwardfacing conformation. Nat Struct Mol Biol 2016, doi: 10.1038/nsmb.3346. 
* 15. Meeske AJ, Riley EP, Robins WP, Uehara T, Mekalanos JJ, Kahne D, Walker S, Kruse AC, Bernhardt TG, Rudner DZ: SEDS proteins are a widespread family of bacterial cell wall polymerases. Nature 2016, 537:634-638.

\section{See comment at Reference No. 11.}

16. Leclercq S, Derouaux A, Olatunji S, Fraipont F, Egan AJF, Vollmer W, Breukink E, Terrak M: Interplay between Penicillin-binding proteins and SEDS proteins promotes bacterial cell wall synthesis. Scientific Report, accepted.

17. Bernhardt TG, de Boer PA: Screening for synthetic lethal mutants in Escherichia coli and identification of EnvC (YibP) as a periplasmic septal ring factor with murein hydrolase activity. Mol Microbiol 2004, 52:1255-1269.

18. Uehara T, Dinh T, Bernhardt TG: LytM-Domain Factors Are Required for Daughter Cell Separation and Rapid Ampicillin-Induced Lysis in Escherichia coli. J Bacteriol 2009, 191:5094-5107.

19. Uehara T, Parzych KR, Dinh T, Bernhardt TG: Daughter cell separation is controlled by cytokinetic ring-activated cell wall hydrolysis. EMBO J 2010, 29:1412-1422.

20. Sauvage E, Kerff F, Terrak M, Ayala JA, Charlier P: The penicillin-binding proteins: structure and role in peptidoglycan biosynthesis. FEMS Microbiol Rev 2008, 32:234-258.

21. Egan AJ, Vollmer W: The physiology of bacterial cell division. Ann N Y Acad Sci 2013, 1277:828.

** 22. Bisson-Filho AW, Hsu YP, Squyres G, Kuru E, Wu F, Jukes C, Dekker C, Holden S, VanNieuwenhze M, Brun Y, et al.: Treadmilling by FtsZ filaments drives peptidoglycan synthesis and bacterial cell division. bioRxiv 2016. doi: http://dx.doi.org/10.1101/077560. Shows together with Reference 29 the treadmilling of FtsZ at the division site. Provides also evidence that the rotation of FtsZ is required for the movement of an essential PG synthase and that septal PG synthesis processive. 
* 23. Morgenstein RM, Bratton BP, Nguyen JP, Ouzounov N, Shaevitz JW, Gitai Z: RodZ links MreB to cell wall synthesis to mediate MreB rotation and robust morphogenesis. Proc Natl Acad Sci U S A 2015, 112:12510-12515.

Shows together with Reference 30 that MreB (dynamics) is required for robust maintainance of rod-shape.

24. van Teeffelen S, Wang S, Furchtgott L, Huang KC, Wingreen NS, Shaevitz JW, Gitai Z: The bacterial actin MreB rotates, and rotation depends on cell-wall assembly. Proc Natl Acad Sci U S A 2011, 108:15822-15827.

25. Fenton AK, Gerdes $\mathrm{K}$ : Direct interaction of FtsZ and MreB is required for septum synthesis and cell division in Escherichia coli. EMBO J 2013, 32:1953-1965.

26. Natale P, Pazos M, Vicente M: The Escherichia coli divisome: born to divide. Environ Microbiol 2013, 15:3169-3182.

27. Bertsche U, Kast T, Wolf B, Fraipont C, Aarsman ME, Kannenberg K, von Rechenberg M, Nguyen-Disteche M, den Blaauwen T, Höltje JV, et al.: Interaction between two murein (peptidoglycan) synthases, PBP3 and PBP1B, in Escherichia coli. Mol Microbiol 2006, 61:675-690.

28. de Pedro MA, Quintela JC, Höltje JV, Schwarz H: Murein segregation in Escherichia coli. J Bacteriol 1997, 179:2823-2834.

** 29. Yang X, Lyu Z, Miguel A, McQuillen R, Huang KC, Xiao J: GTPase activity-coupled treadmilling of the bacterial tubulin FtsZ organizes septal cell-wall synthesis. bioRxiv 2016. doi: http://dx.doi.org/10.1101/077610.

See comment at Reference No. 22.

* 30. Lee TK, Tropini C, Hsin J, Desmarais SM, Ursell TS, Gong EH, Gitai Z, Monds RD, Huang KC: A dynamically assembled cell wall synthesis machinery buffers cell growth. Proc Natl Acad Sci U S A 2014, 111:4554-4559. 
31. Cho H, Wivagg CN, Kapoor M, Barry Z, Rohs PD, Suh H, Marto JA, Garner EC, Bernhardt TG: Bacterial cell wall biogenesis is mediated by SEDS and PBP polymerase families functioning semi-autonomously. Nat Microbiol 2016, 1:16172.

32. Lee TK, Meng K, Shi H, Huang KC: Single-molecule imaging reveals modulation of cell wall synthesis dynamics in live bacterial cells. Nat Commun 2016, 7:13170.

33. Banzhaf M, van den Berg van Saparoea B, Terrak M, Fraipont C, Egan A, Philippe J, Zapun A, Breukink E, Nguyen-Disteche M, den Blaauwen T, et al:: Cooperativity of peptidoglycan synthases active in bacterial cell elongation. Mol Microbiol 2012, 85:179-194.

34. Yousif SY, Broome-Smith JK, Spratt BG: Lysis of Escherichia coli by beta-lactam antibiotics: deletion analysis of the role of penicillin-binding proteins $1 \mathrm{~A}$ and 1B. J Gen Microbiol 1985, 131:2839-2845.

35. Garcia del Portillo F, de Pedro MA: Differential effect of mutational impairment of penicillinbinding proteins $1 \mathrm{~A}$ and $1 \mathrm{~B}$ on Escherichia coli strains harboring thermosensitive mutations in the cell division genes fts $A$, fts $Q, f t s Z$, and pbpB. J Bacteriol 1990, 172:58635870.

36. Müller P, Ewers C, Bertsche U, Anstett M, Kallis T, Breukink E, Fraipont C, Terrak M, NguyenDistèche M, Vollmer W: The essential cell division protein FtsN interacts with the murein (peptidoglycan) synthase PBP1B in Escherichia coli. J Biol Chem 2007, 282:36394-36402.

* 37. Gray AN, Egan AJ, Van't Veer IL, Verheul J, Colavin A, Koumoutsi A, Biboy J, Altelaar AF, Damen MJ, Huang KC, et al.: Coordination of peptidoglycan synthesis and outer membrane constriction during Escherichia coli cell division. eLife 2015, 4. Identified functional interactions between the septal PG synthase PBP1B and two members of the Tol complex to coordinate PG synthesis with outer membrane constriction.

38. Derouaux A, Wolf B, Fraipont C, Breukink E, Nguyen-Distèche M, Terrak M: The monofunctional glycosyltransferase of Escherichia coli localizes to the cell division site 
and interacts with penicillin-binding protein 3, FtsW, and FtsN. J Bacteriol 2008, 190:1831-1834.

39. Magnet S, Dubost L, Marie A, Arthur M, Gutmann L: Identification of the L,D-transpeptidases for peptidoglycan cross-linking in Escherichia coli. J Bacteriol 2008, 190:4782-4785.

40. Magnet S, Bellais S, Dubost L, Fourgeaud M, Mainardi JL, Petit-Frere S, Marie A, MenginLecreulx D, Arthur M, Gutmann L: Identification of the L,D-transpeptidases responsible for attachment of the Braun lipoprotein to Escherichia coli peptidoglycan. J Bacteriol 2007, 189:3927-3931.

41. Heidrich C, Ursinus A, Berger J, Schwarz H, Höltje JV: Effects of multiple deletions of murein hydrolases on viability, septum cleavage, and sensitivity to large toxic molecules in Escherichia coli. J Bacteriol 2002, 184:6093-6099.

42. Singh SK, Parveen S, SaiSree L, Reddy M: Regulated proteolysis of a cross-link-specific peptidoglycan hydrolase contributes to bacterial morphogenesis. Proc Natl Acad Sci U S A 2015, 112:10956-10961.

43. Nelson DE, Young KD: Penicillin-binding protein 5 affects cell diameter, contour, and morphology of Escherichia coli. J Bacteriol 2000, 182:1714-1721.

44. Nelson DE, Young KD: Contributions of PBP 5 and DD-carboxypeptidase penicillin binding proteins to maintenance of cell shape in Escherichia coli. J Bacteriol 2001, 183:3055-3064.

45. Rosenbusch JP: Structural and functional properties of porin channels in $E$. coli outer membranes. Experientia 1990, 46:167-173.

** 46. Peters K, Kannan S, Rao VA, Biboy J, Vollmer D, Erickson SW, Lewis RJ, Young KD, Vollmer W: The Redundancy of Peptidoglycan Carboxypeptidases Ensures Robust Cell Shape Maintenance in Escherichia coli. MBio 2016, 7.

This work revealed the important function of a previously considered minor DD-CPase, PBP6b in E. coli growing at low $\mathrm{pH}$ and, hence, this paper shows that seemingly redundant PG hydrolases can have important roles at certain growth conditions. 
380 47. Lommatzsch J, Templin MF, Kraft AR, Vollmer W, Höltje JV: Outer membrane localization of murein hydrolases: MItA, a third lipoprotein lytic transglycosylase in Escherichia coli. J Bacteriol 1997, 179:5465-5470.

383

384 
Table 1: Essential enzymes and redundancies in the PG synthesis pathway of E. coli. See main text for discussion and references.

\begin{tabular}{|c|c|}
\hline Function & Proteins \\
\hline $\begin{array}{l}\text { Intracellular } \\
\text { lipid II } \\
\text { synthesis }\end{array}$ & $\begin{array}{l}\text { Essential enzymes: MurA; MurB; MurC; MurC; MurD; MurE; MurF; } \\
\text { MurI; MraY; MurG } \\
\text { - Redundancy in essential steps: Alr, DadX (alanine racemases); D- } \\
\text { DdlA, DdlB (alanyl-D-alanine ligases) }\end{array}$ \\
\hline PG synthesis & $\begin{array}{l}\text { Essential class B PBP - SEDS pairs for different processes: PBP2- } \\
\text { RodA (cell elongation); PBP3-FtsW (cell division) } \\
\text { - Semi-redundancy in class A PBP PG synthases - activator function: } \\
\text { PBP1A - LpoA; PBP1B - LpoB } \\
\text { - Non-essential components: PBP1C, MtgA }\end{array}$ \\
\hline $\begin{array}{l}\text { LD-trans- } \\
\text { peptidases }\end{array}$ & $\begin{array}{l}\text { - Multiple enzymes for LD-crosslink formation: YnhG, YcbB } \\
\text { - Multiple enzymes for lipoprotein attachment: ErfK, YbiS, YcfS }\end{array}$ \\
\hline PG hydrolysis & $\begin{array}{l}\text { Multiple non-essential lytic transglycosylases: Slt, MltA, MltB, MltC, } \\
\text { MltD, MltE, MltF, MltG } \\
\text { Multiple non-essential DD-endopeptidases: PBP4, PBP7, MepA, MepS, } \\
\text { MepM, MepH } \\
\text { Multiple non-essential DD-carboxypeptidases: PBP4, PBP4b, PBP5, } \\
\text { PBP6a, PBP6b, PBP7, AmpH } \\
\text { DD-endopeptidases that are redundantly essential for cell elongation: } \\
\text { MepS, MepM, MepH } \\
\text { - Redundant MurNAc-L-Ala amidases - activators for cell separation: } \\
\text { AmiA - EnvC; AmiB - EnvC; AmiC - NlpD } \\
\text { Semi-redundant DD-carboxypeptidases to maintain cell shape at } \\
\text { different pH values: PBP5; PBP6b }\end{array}$ \\
\hline
\end{tabular}


Figure 1. Theoretical minimal system of PG growth [20]. The GTase domain of a class A PBPs (yellow) utilizes lipid II (red) to synthesize a glycan chain, which is incorporated into the existing PG layer by the TPase domains of class A and B PBPs (grey and purple), while an EPase (green) cleaves in a cross-link in the PG to allow the insertion of the new glycan chain. Alternatively, the system could work with only one TPase that is flexible enough to utilize peptides from different glycan chains.

Figure 2. Model for growth condition-dependent composition of dynamic complexes. In non-

growing cells and in the absence of PG synthesis single molecules or partial complexes of PG enzymes, their regulators and cytoskeletal elements (represented as colored circles) diffuse in the cell membrane or periplasm as indicated by the small arrows (upper part). Protein redundancy is indicated by different colors but for simplicity only two colors were used for each category of PG enzymes and their regulators, although in the cell the level of redundancy is higher. Dynamic PG-synthesizing complexes (enclosed by dashed circles) assemble for rounds of PG synthesis at the side wall (for cell elongation) or mid-cell (for cell division) (middle and bottom part), and active complexes move processively in the direction of glycan chains (large arrows). The composition of the active complexes depends on the growth condition, which affects the properties of the periplasm (left side). For example, growth condition A (blue color) could stand for growth at $\mathrm{pH} 7.5$ and growth condition B for growth at $\mathrm{pH}$ 5. Other growth variables such as osmolality, ion strength and temperature can also affect the composition of the PG synthesis complexes. OM, outer membrane; PG, peptidoglycan layer; IM, inner membrane. 


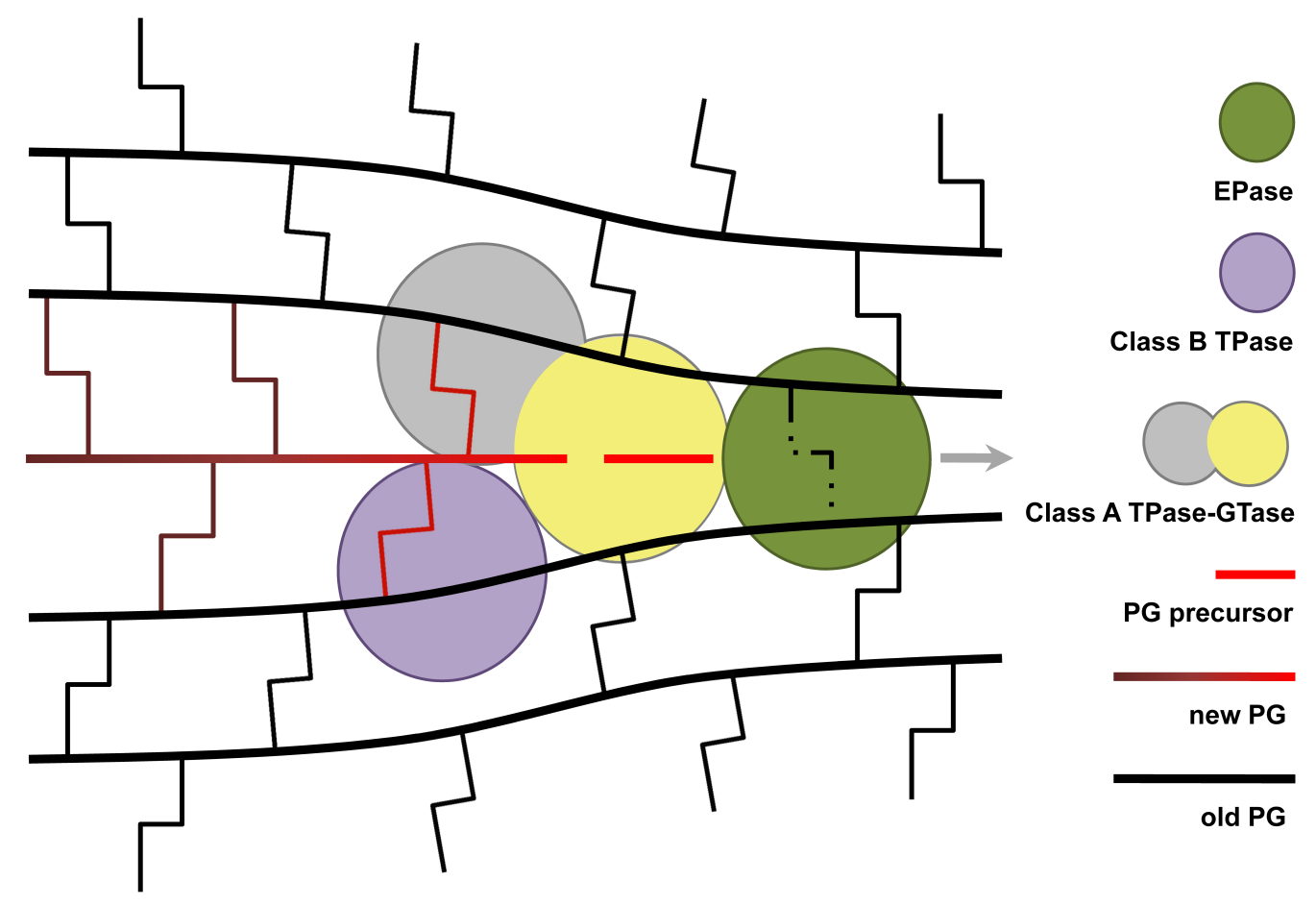




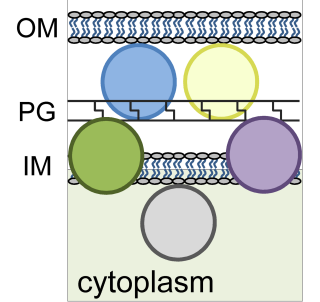

Growth media A

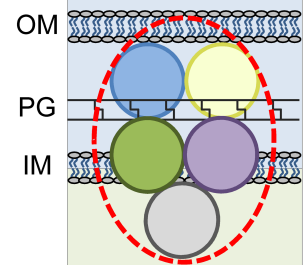

cytoplasm

Growth media B

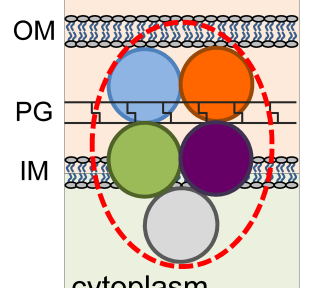

cytoplasm

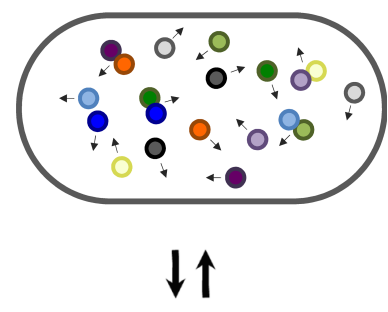

Elongation

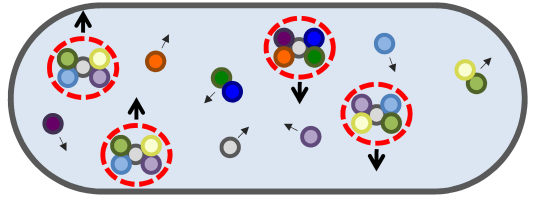

$\downarrow \uparrow$
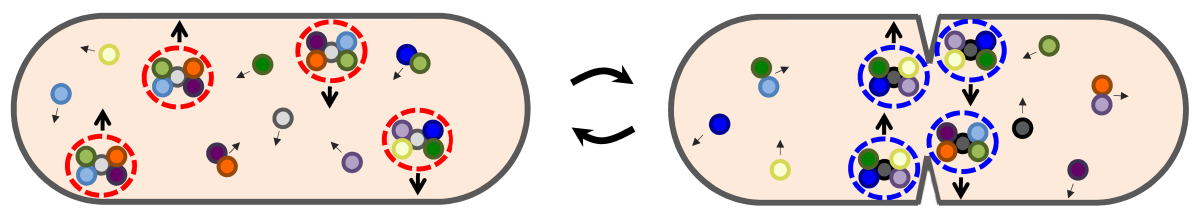

Growth condition B

Septation

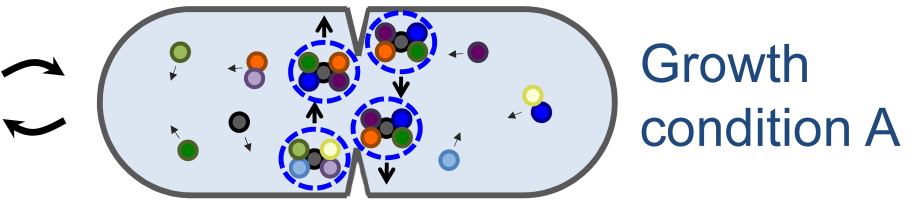

$\downarrow \uparrow$
PG synthases

$\bigcirc$ Regulators of synthases

$\bigcirc \quad$ MreB and regulators
PG hydrolases

0 Regulators of hydrolases

FtsZ and regulators 